\title{
A rare case of posttraumatic bilateral orbital myositis in a young boy — a case report
}

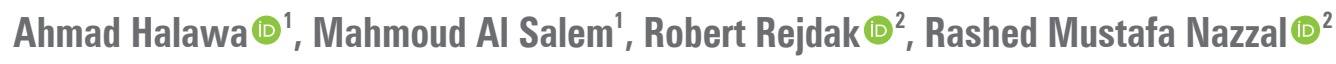 \\ 'Ilbn Al Haytham Hospital, Amman, Jordan \\ ${ }^{2}$ Medical University of Lublin, Lublin, Poland
}

\begin{abstract}
Orbital myositis $(\mathrm{OM})$ is a benign inflammatory disease of the orbit characterised by a polymorphous lymphoid infiltrate with varying degrees of fibrosis, without a known local or systemic cause. In this paper, we present a case of a young boy who sustained a trauma to his eyes a few days prior to admission, after which he developed bilateral orbital pain and ocular motility limitation. He underwent the appropriate investigations including orbital imaging and blood laboratory workup, which were all consistent with a diagnosis of posttraumatic bilateral orbital myositis. He was treated with steroids for few weeks, and when the dose of steroids was tapered, he had a relapse of the same disease with a different presentation, which was later controlled with a higher dose of steroids, after which the patient went into remission.
\end{abstract}

KEY WORDS: myositis; steroids; orbit; trauma

Ophthalmol J 2020; Vol. 5, 1-4

\section{INTRODUCTION}

Idiopathic orbital inflammatory disease (IOID) is the term used to describe the disease with an idiopathic cause, spontaneous resolution, benign, non-infectious, space-occupying lesion typically presenting in the third to sixth decade of life with non-granulomatous changes on histopathology. Although orbital myositis is the most common presentation of this disease, other subtypes includes dacryoadenitis, perineuritis, eyelid pseudotumour, inflammation of the anterior orbit (e.g. scleritis), and diffuse orbital inflammation [1].

While IOID is the third most common cause of unilateral proptosis in adults after thyroid eye disease and lymphoproliferative disease [2], it is a relatively rare disease in children. Idiopathic orbital inflammatory disease is a diagnosis of exclusion, hence a wide set of differentials should be considered and ruled out before labelling a patient with this diagnosis, such as thyroid eye disease, lymphoproliferative disease, rhabdomyosarcoma, orbital cellulitis, and metastatic conditions. In this case report, we ruled out all other differential diagnoses by the appropriate laboratory and imaging techniques, and the diagnosis of orbital myositis was made.

\section{CASE PRESENTATION}

A 12-year-old boy, previously healthy, presented to our clinic with acute diplopia of two days' duration. The patient's history dates back to 10 days prior to presentation, when he sustained a trauma to his face with a football while playing football. On the next morning he started complaining of bilateral eyelid swelling. Over the following few days he developed deep aching pain in both eyes, associated with generalised fatigue and vomiting. 


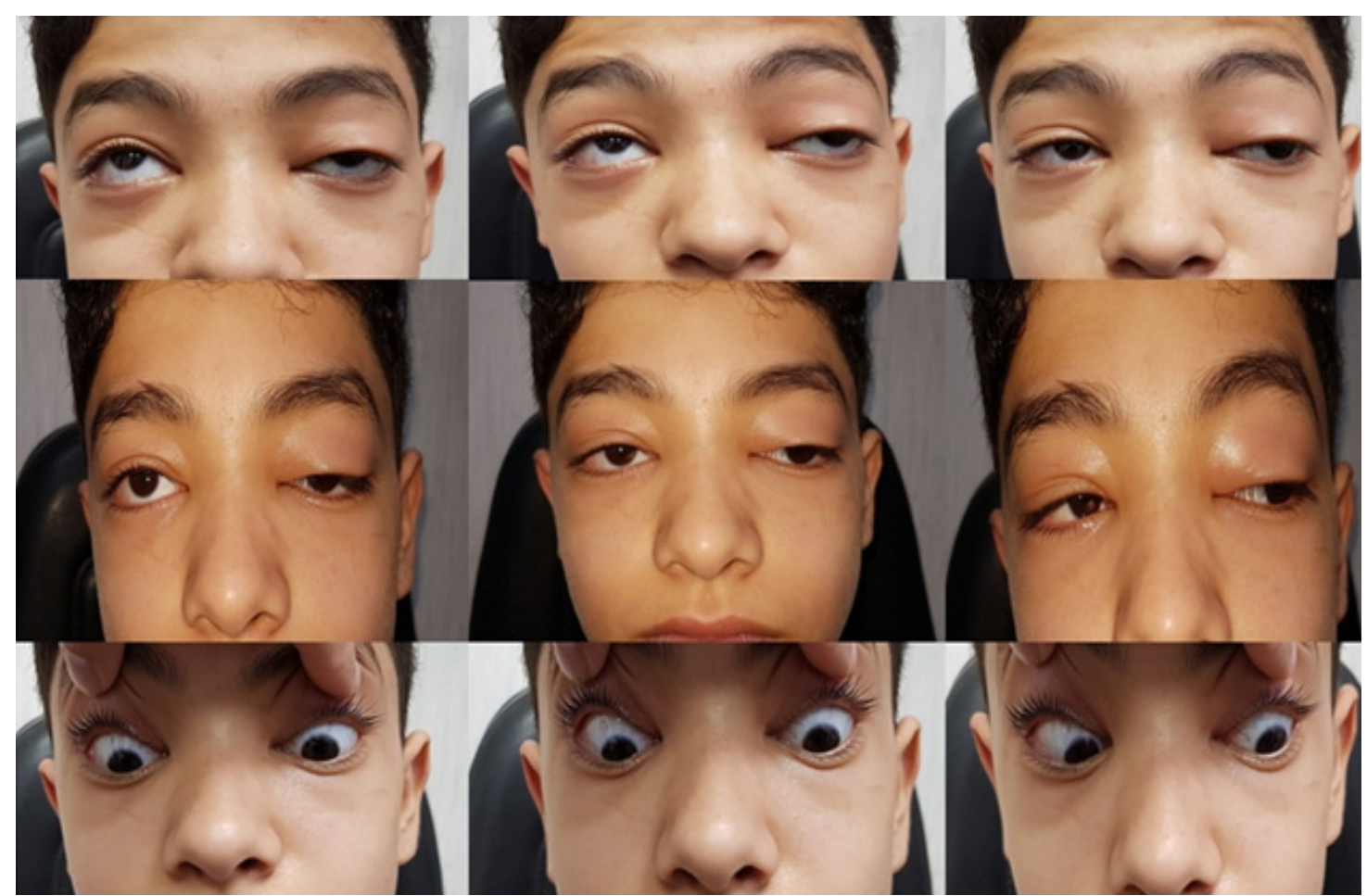

FIGURE 1. Ocular motility at presentation showing limited right abduction and left adduction

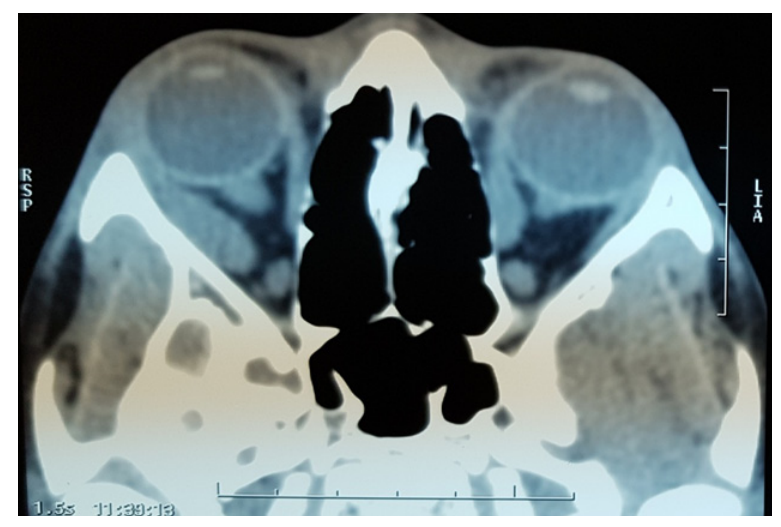

FIGURE 2. Orbital CT scan showing enlarged muscle belly including the tendon (mainly right lateral rectus and left medial rectus)

He eventually developed binocular diplopia. On examination, there was a bilateral eyelid swelling. Anterior and posterior segments exam was within normal limits. Ocular motility exam showed a profound limitation of abduction in the right eye and to a lesser extent limitation of adduction and elevation in the left eye (Fig. 1).

The patient was admitted for further evaluation, and a preliminary diagnosis of orbital cellulitis was suspected, so he was started on intravenous antibiotics.
Orbital CT scan (Fig. 2) revealed prominent enlarged muscles, involving the muscle tendon, mainly the lateral rectus, and superior rectus on the right side and medial rectus and superior rectus on the left side. Blood workup was obtained and included the following: complete blood count, erythrocyte sedimentation rate (ESR), C-reactive protein (CRP), thyroid function test, and workup for connective tissue diseases [rheumatoid factor (RF), antinuclear antibody (ANA), and C- and Pantineutrophil cytoplasmic antibodies (ANCA)], and all were within normal limits.

The diagnosis of IOID was made and the patient was switched from antibiotics to intravenous methylprednisolone $(250 \mathrm{mg}$ per day, given slowly with close monitoring over three hours) for three days, after which the patient showed dramatic improvement in symptoms and ocular motility (Fig. 3).

The patient was discharged on oral prednisolone $(1 \mathrm{mg} / \mathrm{kg}$ ) with slow tapering over six weeks. The patient was doing well until he reached the last week of tapering, when he started complaining of recurrence of his deep aching orbital pain with right-eye redness. On examination, there was right episcleritis (Fig. 4) and restriction of ocular motility (Fig. 5). Surprisingly, contrary to what he presented with, this time right abduction was normal and right ad- 


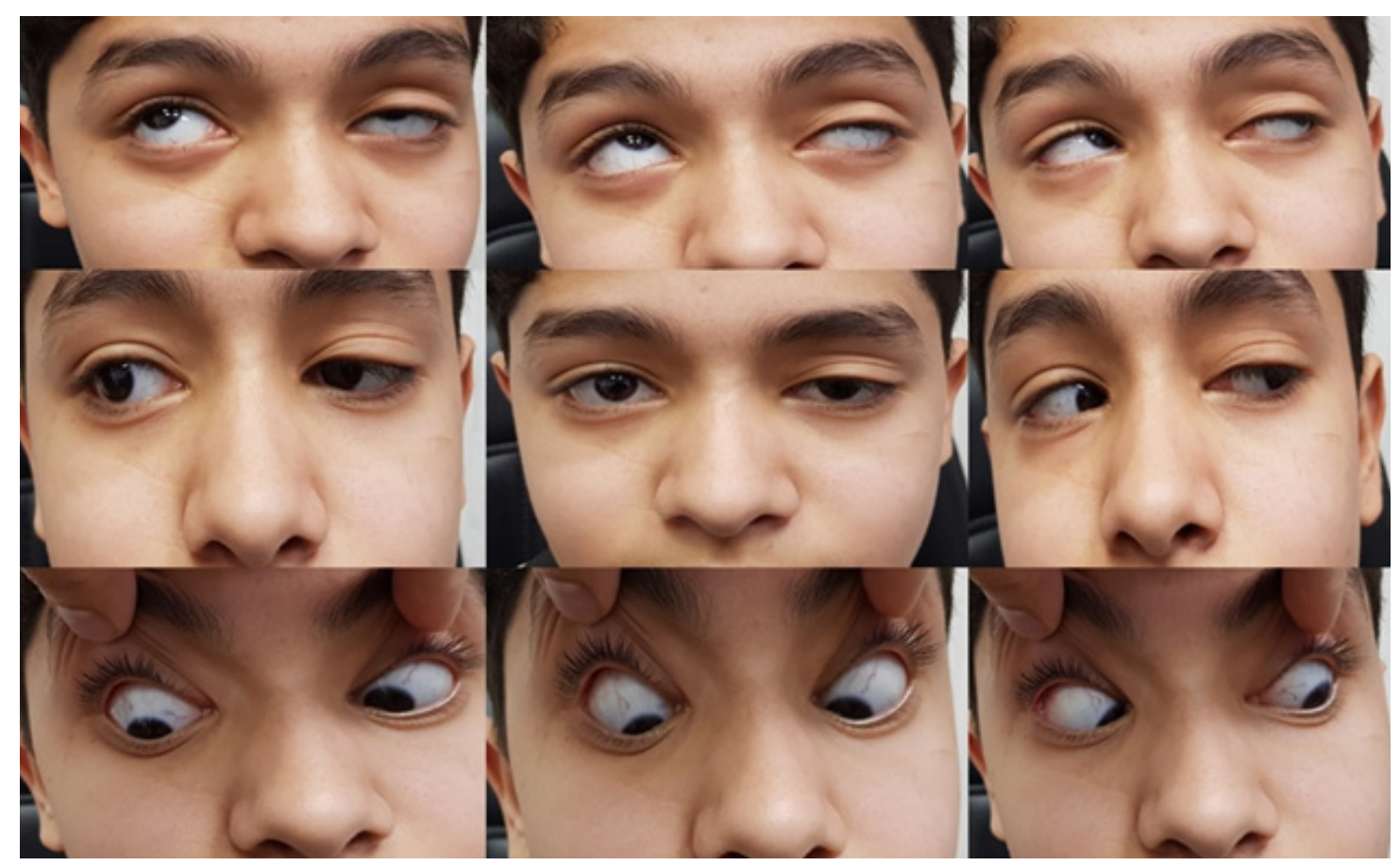

FIGURE 3. Ocular motility after improvement

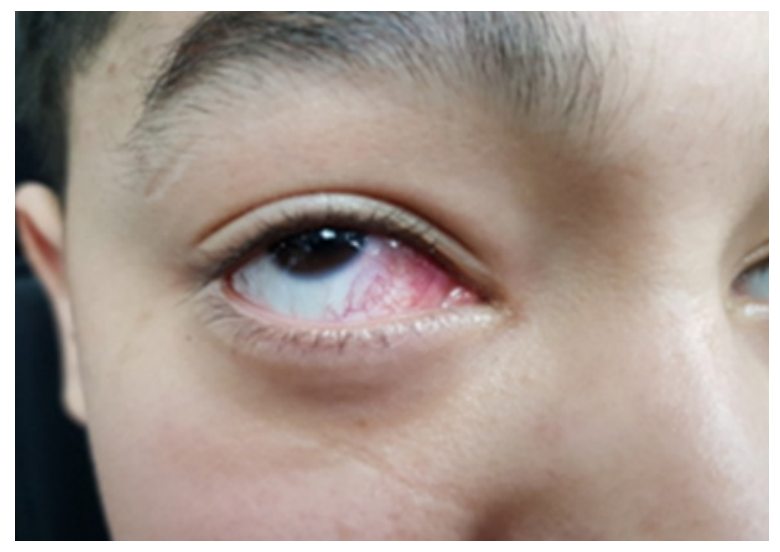

FIGURE 4. Right episcleritis

duction was restricted. The patient was started again on oral prednisolone with a longer tapering period and oral azathioprine as a steroid sparing agent (the dose and the appropriate follow-up were in liaison with a paediatrician). The patient was followed-up for few months, during which he remained in remission even after discontinuation of medications.

\section{DISCUSSION}

Nonspecific orbital inflammation was first described in 1905 by Birch-Hirschfeld. Later on, it was named as inflammatory pseudotumor in

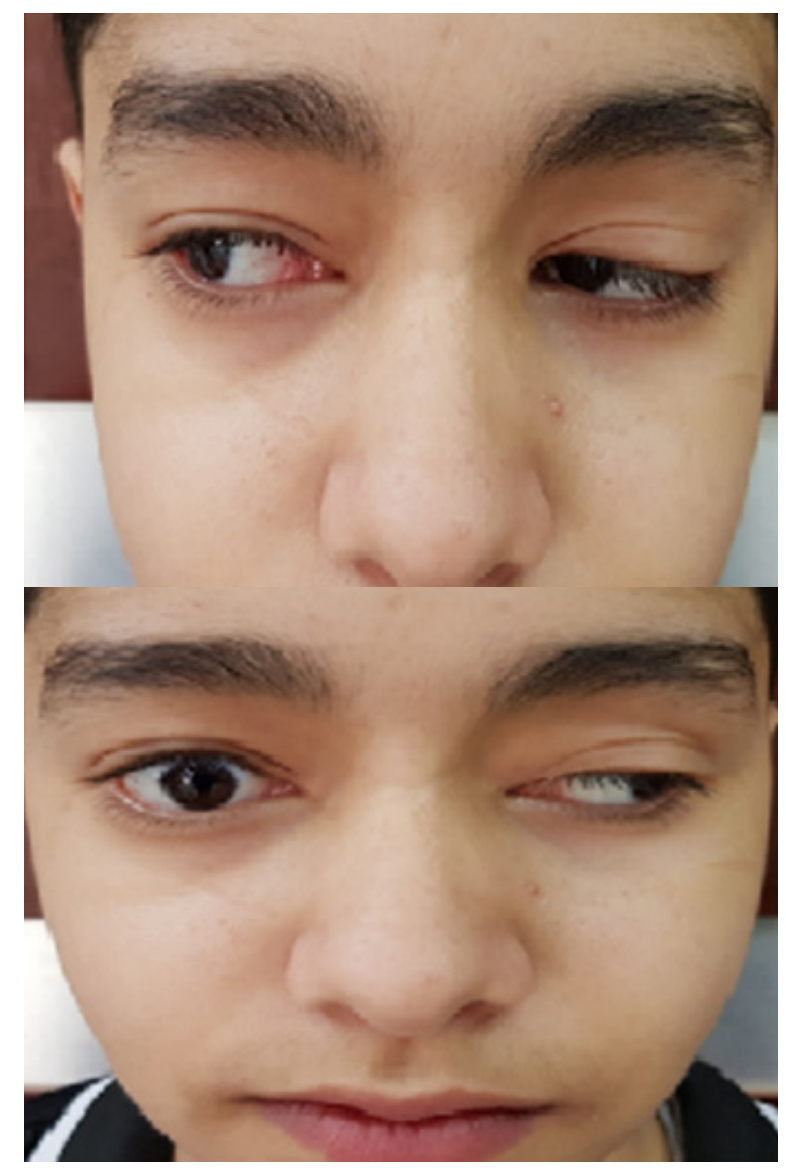

FIGURE 5. Right gaze and left gaze showing limited left adduction on disease recurrence 
1954 by Umiker et al. because of its propensity to mimic a malignant process. Presently, the term IOID is usually used. While IOID may account for $5 \%$ to $16 \%$ of all orbital lesions in adults, it is rare in children, causing a diagnostic dilemma [3]. The pathogenesis of the disease remains controversial; both infectious and immune-mediate aetiologies have been implicated, and association with upper respiratory tract infections and viral illnesses has been described [4, 5]. Of interest, Mottow-Lippe et al. suggested that trauma may cause increased vascular permeability, resulting in release of antigenic substances, which in turn incite an inflammatory cascade [6]. Hence, we believe that trauma was the factor triggering the inflammation in our patient.

The symptoms and clinical findings in IOID may vary widely, but they are dictated by the degree and anatomical location of the inflammation. It can present with deep-rooted boring pain, eyelid swelling, conjunctival injection, ptosis, proptosis, diplopia, and restriction of ocular motility. Affected children more often have constitutional signs and symptoms, such as headache, fever, malaise, emesis, anorexia, lethargy, and abdominal pain [7].

Because IOID is a diagnosis of exclusion, a broad workup is usually done to exclude other causes. The differential diagnosis of idiopathic OM includes the following: infections (viral infections, orbital cellulitis, orbital abscess, Lyme disease, herpes zoster, and syphilis), inflammatory reaction (trauma, foreign body, bisphosphonate-related reaction, and postvaccinal reaction), Tolosa-Hunt syndrome, thyroid ophthalmopathy, vasculitis (Wegener's granulomatosis, polyarteritis nodosa, rheumatoid arteritis, giant cell arteritis, and Kawasaki disease), systemic lupus erythematosus, sarcoidosis, inflammatory bowel disease, neoplasm, arteriovenous fistulas, and malformations [8]. On radiologic imaging of idiopathic orbital myositis, there is diffuse muscle enlargement involving the muscle tendon, in contrast to thyroid orbitopathy in which the tendons are spared. In addition, in idiopathic orbital myositis, the lateral rectus and medial rectus are the most likely to be involved, while in thyroid disease the lateral rectus is the last rectus muscle to be affected [9].

Systemic corticosteroid therapy is the mainstay treatment in the management of IOID [10]. Of diagnostic significance, idiopathic orbital inflammation is characterised by a rapid and favourable response to systemic corticosteroid treatment.
This disease condition in our patient demonstrated some rare and unusual characteristics, such as bilateral eye involvement in a post-traumatic presentation and ophthalmoplegia - from mainly involving the right lateral rectus at presentation to involving the right medial rectus on disease recurrence.

\section{CONCLUSION}

Orbital myositis, as the most important subtype of IOID, is a rare diagnosis that should be kept in mind in patients presenting with ocular pain or diplopia after an ocular trauma. Being a diagnosis of exclusion, IOID should be considered after adequate imaging and laboratory investigations have been performed.

\section{Acknowledgements}

None

\section{Statement of competing interests}

No competing interests.

\section{REFERENCES}

1. Grouteau $E$, Chaix $Y$, Armbruster V. [Idiopathic inflammatory orbital myositis and idiopathic inflammatory pseudotumor in children: three cases]. Arch Pediatr. 1998; 5(2): 153-158, doi: 10.1016/s0929693x(97)86829-7, indexed in Pubmed: 10223136.

2. Belanger $\mathrm{C}$, Zhang KS, Reddy AK, et al. Inflammatory disorders of the orbit in childhood: a case series. Am J Ophthalmol. 2010; 150(4): 460463, doi: 10.1016/j.ajo.2010.05.012, indexed in Pubmed: 20688313.

3. Mulvihill A, Smith CR, Buncic JR. Pediatric orbital pseudotumour presenting as a painless orbital and periocular mass. Can $\mathrm{J}$ Ophthalmol. 2004; 39(1): 77-79, doi: 10.1016/s0008-4182(04)80057-7, indexed in Pubmed: 15040619.

4. Yuen SJ, Rubin PA. Idiopathic orbital inflammation: ocular mechanisms and clinicopathology. OphthalmolClin North Am. 2002; 15(1): 121-6, doi: 10.1016/s0896-1549(01)00003-7, indexed in Pubmed: 12064075.

5. Espinoza GM. Orbital inflammatory pseudotumors: etiology, differential diagnosis, and management. Curr Rheumatol Rep. 2010; 12(6): 443447, doi: 10.1007/s11926-010-0128-8, indexed in Pubmed: 20803107.

6. Mottow-Lippa L, Jakobiec FA, Smith M. Idiopathic inflammatory orbital pseudotumor in childhood. II. Results of diagnostic tests and biopsies. Ophthalmology. 1981; 88(6): 565-574, doi: 10.1016/s01616420(81)34984-7, indexed in Pubmed: 7267028.

7. Stevens JL, Rychwalski PJ, Baker RS, et al. Pseudotumor of the orbit in early childhood. J AAPOS. 1998; 2(2): 120-123, doi: 10.1016/ s1091-8531(98)90076-3, indexed in Pubmed: 10530975.

8. Benmiloud S, Boubbou M, Hida M. Bilateral idiopathic orbital myositis in an infant. Int J Res Med. 2013; 2: 112-114.

9. Schoser BG. Ocular myositis: diagnostic assessment, differential diagnoses, and therapy of a rare muscle disease - five new cases and review. ClinOphthalmol. 2007; 1(1): 37-42, indexed in Pubmed: 19668464 .

10. Hankey GJ, Silbert PL, Edis RH, et al. Orbital myositis: a study of six cases. Aust N Z J Med. 1987; 17(6): 585-591, doi: 10.1111/j.14455994.1987.tb01264.x, indexed in Pubmed: 3328976. 